\title{
BMJ Open How accurate are digital symptom assessment apps for suggesting conditions and urgency advice? A clinical vignettes comparison to GPs
}

Stephen Gilbert (D) , ${ }^{1}$ Alicia Mehl, ${ }^{1}$ Adel Baluch, ${ }^{1}$ Caoimhe Cawley, ${ }^{1}$ Jean Challiner, ${ }^{1}$ Hamish Fraser, ${ }^{2}$ Elizabeth Millen, ${ }^{1}$ Maryam Montazeri (D) , ${ }^{1}$ Jan Multmeier, ${ }^{1}$ Fiona Pick, ${ }^{1}$ Claudia Richter, ${ }^{1}$ Ewelina Türk, ${ }^{1}$ Shubhanan Upadhyay, ${ }^{1}$ Vishaal Virani, ${ }^{1}$ Nicola Vona, ${ }^{1}$ Paul Wicks, ${ }^{1}$ Claire Novorol ${ }^{1}$

To cite: Gilbert S, Mehl A, Baluch A, et al. How accurate are digital symptom assessment apps for suggesting conditions and urgency advice? A clinical vignettes comparison to GPs. BMJ Open 2020;10:e040269. doi:10.1136/ bmjopen-2020-040269

- Prepublication history and additional materials for this paper is available online. To view these files, please visit the journal online (http://dx.doi. org/10.1136/bmjopen-2020040269).

SG and $A M$ contributed equally.

Received 11 May 2020

Revised 27 0ctober 2020

Accepted 16 November 2020

Check for updates

(C) Author(s) (or their employer(s)) 2020. Re-use permitted under CC BY-NC. No commercial re-use. See rights and permissions. Published by BMJ.

${ }^{1}$ Ada Health GmbH, Berlin, Germany

${ }^{2}$ Brown Center for Biomedical Informatics, Brown University, Rhode Island, USA

Correspondence to Dr Stephen Gilbert; science@ada.com

\section{ABSTRACT}

Objectives To compare breadth of condition coverage, accuracy of suggested conditions and appropriateness of urgency advice of eight popular symptom assessment apps.

Design Vignettes study.

Setting 200 primary care vignettes.

Intervention/comparator For eight apps and seven general practitioners (GPs): breadth of coverage and condition-suggestion and urgency advice accuracy measured against the vignettes' gold-standard.

Primary outcome measures (1) Proportion of conditions 'covered' by an app, that is, not excluded because the user was too young/old or pregnant, or not modelled; (2) proportion of vignettes with the correct primary diagnosis among the top 3 conditions suggested; (3) proportion of 'safe' urgency advice (ie, at gold standard level, more conservative, or no more than one level less conservative). Results Condition-suggestion coverage was highly variable, with some apps not offering a suggestion for many users: in alphabetical order, Ada: 99.0\%; Babylon: 51.5\%; Buoy: 88.5\%; K Health: 74.5\%; Mediktor: 80.5\%; Symptomate: 61.5\%; Your.MD: 64.5\%; WebMD: 93.0\%. Top-3 suggestion accuracy was GPs (average): 82.1\% $\pm 5.2 \%$; Ada: $70.5 \%$; Babylon: $32.0 \%$; Buoy: 43.0\%; K Health: 36.0\%; Mediktor: 36.0\%; Symptomate: 27.5\%; WebMD: 35.5\%; Your.MD: 23.5\%. Some apps excluded certain user demographics or conditions and their performance was generally greater with the exclusion of corresponding vignettes. For safe urgency advice, tested GPs had an average of $97.0 \% \pm 2.5 \%$. For the vignettes with advice provided, only three apps had safety performance within $1 \mathrm{SD}$ of the GPs—Ada: $97.0 \%$; Babylon: 95.1\%; Symptomate: 97.8\%. One app had a safety performance within 2 SDs of GPs_-Your.MD: $92.6 \%$. Three apps had a safety performance outside 2 SDs of GPs—Buoy: $80.0 \%(p<0.001)$; K Health: $81.3 \%(p<0.001)$; Mediktor: $87.3 \%\left(p=1.3 \times 10^{-3}\right)$.

Conclusions The utility of digital symptom assessment apps relies on coverage, accuracy and safety. While no digital tool outperformed GPs, some came close, and the nature of iterative improvements to software offers scalable improvements to care.
Strengths and limitations of this study

- The study included a large number of vignettes which were peer reviewed by independent and experienced primary care physicians to minimise bias.

- General practitioners and apps were tested with vignettes in a manner that simulates real clinical consultations.

- Detailed source data verification was carried out.

- Vignette entry was conducted by professionals as a recent study found that laypeople are less good at entering vignettes for symptoms that they have never experienced.

- Limitations include the lack of a rigorous and comprehensive selection process to choose the eight apps and the lack of real patient experience assessment.

\section{INTRODUCTION}

Against the background of an ageing population and rising pressure on medical services, the last decade has seen the internet replace general practitioners (GPs) as the first port of call for health information. A 2010 survey of over 12000 people from 12 countries reported that $75 \%$ of respondents search for health information online, ${ }^{1}$ with some twothirds of patients in 2017 reporting that they 'google' their symptoms before going to the doctor's office. ${ }^{2}$ However, online search tools like Google or Bing were not intended to provide medical advice and risk offering irrelevant or misleading information. ${ }^{3}$ One potential solution is dedicated symptom assessment applications (ie, apps), ${ }^{3-6}$ which use a structured interview or multiple-choice format to ask patients questions about their demographic, relevant medical history, symptoms, and presentation. In the first few screening questions, some symptom assessment apps 
exclude patients from using the tool if they are too young, too old, are pregnant, or have certain comorbidities, limiting the 'coverage' of the tool. Exclusion limits the range of users for whom the app can be turned to for advice, but, depending on the market segment the app manufacturer wants to address, having a narrow coverage may be appropriate, and it may in certain circumstances have advantages, for example, if it was a requirement of a regulatory authority within a certain jurisdiction, or, if it was possible to design the app with greater usability by narrowing its focus. Assuming the patient is not excluded, these software tools use a range of computational approaches to suggest one or more conditions that might explain the symptoms (eg, common cold vs pneumonia). Many symptom assessment apps then suggest next steps that patients should take (levels of urgency advice, for example, self-care at home vs seek urgent consultation), often along with evidence-based condition information for the user.

A recent systematic review of the literature identified that rigorous studies are required to show that these apps provide safe and reliable information ${ }^{4}$ in the context for which they were designed and for which they have regulatory approval. Most previous studies considered only a single symptom assessment app, focused on specific (often specialty) conditions, had a small number of vignettes $(<50)$, were relatively uncontrolled in the nature of the cases presented, and suffered a high degree of bias. ${ }^{4}$ For example, a previous study examined the performance of the Mediktor app in the emergency department (ED) waiting room. ${ }^{7}$ While this is a valid setting, most apps were designed and approved for use primarily at home and for newly presenting problems; accordingly, some $38.7 \%$ of patients had to be excluded. Few studies have systematically compared symptom assessment apps to one another in this context, which is particularly important as apps may increasingly be used to supplement or replace telephone triage. ${ }^{4}$ This is particularly relevant in 2020 due to the COVID-19 pandemic - early in the spread of COVID19 , healthcare facilities risked being overwhelmed and furthering contagion, so communication strategies were needed to provide patients with advice without face-toface contact. ${ }^{89}$

In contrast to deploying apps in a heterogeneous realworld setting, where participants would not have the time to re-enter their symptoms multiple times, and may not receive a verifiable diagnosis, clinical vignettes studies allow direct comparison of interapp and app-to-GP performance. ${ }^{10-12}$ Clinical vignettes are created to represent patients, these are reviewed and then assigned goldstandard answers for main and differential diagnoses and for triage. The clinical vignettes are then used to test both apps and GPs. GPs are assessed through mock telephone consultations and apps through their normal question flow. ${ }^{36}$ Clinical vignettes studies have the advantage of enabling direct GP-to-app comparison, allowing a wide range of case types to be explored, and are generalisable to 'real-life' situations, but are complementary to, not a replacement for, real-patient studies. ${ }^{4}{ }^{10}$ Seminal work at Harvard Medical School has established the value of such approaches but has not been updated recently. ${ }^{36}$

The objective of the current study was to compare the coverage, suggested condition accuracy, and urgency advice accuracy of GPs and eight popular symptom assessment apps which provide, for a general population, condition suggestions and urgency advice: Ada, Babylon, Buoy, K Health, Mediktor, Symptomate, WebMD, and Your.MD. We had three primary hypotheses:

1. That GPs would have better performance than the apps in the three metrics of (a) condition suggestion accuracy, (b) appropriateness of urgency advice and (c) safety of urgency advice.

2. That performance of each app would be consistent across the three metrics (condition-suggestion accuracy, appropriateness and safety of urgency advice).

3. That apps would differ from one another in their performance across the three metrics.

Exploration of these hypotheses is important for users of the applications and for physicians.

\section{METHODS}

The process for clinical vignette creation, review and testing of the GPs and the apps using the vignettes is shown in figure 1.

\section{Clinical vignette creation}

An independent primary care clinical expert consultant (JC) was commissioned to lead the creation of 200 clinical vignettes: JC has over 25 years' experience in general practice and emergency practice and has also had many years of experience in creating and customising algorithms for use in telephone triage and for internet-based selfassessment, including for National Health Service, UK (NHS Direct). The vignette creation team also included two GPs (SU and AB-employees of Ada Health), each with over 5 years primary care and $\mathrm{ED}$ experience. $\mathrm{SU}$ and $\mathrm{AB}$ had worked for the Ada Health telehealth service Dr Chat but were not involved in the development of Ada's medical intelligence. The vignettes were designed to include both common and less-common conditions relevant to primary care practice, and to include clinical presentations and conditions affecting all body systems. They were created to be fair cases representing real-world situations in which a member of the public might seek medical information or advice from a symptom assessment app, or present to primary care. Most of the clinical vignettes were newly presenting problems experienced by an individual or by a child in their care, and they included some patients with chronic conditions, for example, diabetes, hypertension, and so on (see online supplemental tables 1-3).

The origin of $32.0 \%$ of the vignettes (numbers 1-64) was anonymised insights from transcripts of real calls made to NHS Direct (a UK national nurse-led telephone next-steps advice/triage service operational until 2014) which had previously been used as part of an NHS Direct 
Vignette creation

Review and answer setting panels
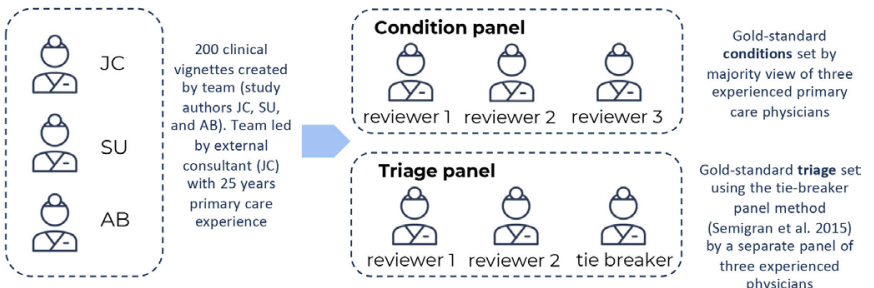

\section{Testing diagnosis and triage tested of GPS $\begin{gathered}\text { with clinical vignettes for } \\ \text { GPs via telephone } \\ \text { consultations with Ic. }\end{gathered}$}

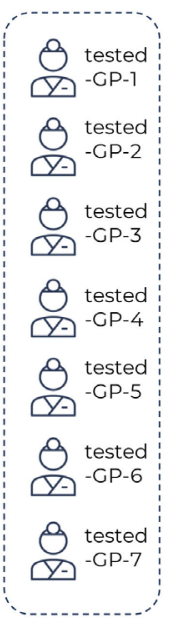

Testing of apps

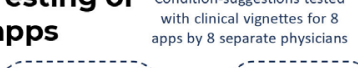

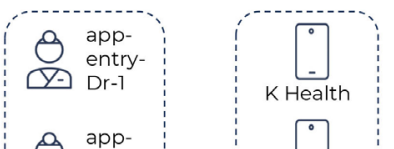
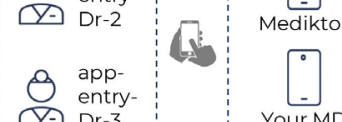
$y$ - Dr-3 8 appDr-4 8 appDr-5

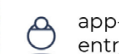
$\forall-1$ Dr-6

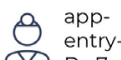
8 entry-7 Mediktor

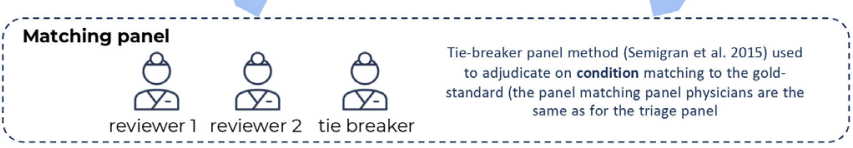

Output

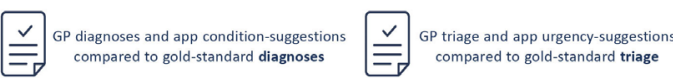

Figure 1 Overview of the study methodology including: (1) vignette creation; (2) vignettes review and answer setting; (3) testing of general practitioners (GPs); and (4) adjudication of matching of condition suggestions to the gold standard.

benchmarking exercise for recommended outcomes (these were used with full consent of NHS Direct). The remaining $68.0 \%$ of the vignettes were created by the vignette creation team $(\mathrm{JC}, \mathrm{SU}$ and $\mathrm{AB})$, including joint assignment of the most appropriate main diagnosis and differential diagnoses, as a starting point for the vignette gold-standard answers. The vignettes included the sex and age of the patient, previous medical history (including factors such as pregnancy, smoking, high blood pressure, diabetes, other illnesses), the named primary complaint, additional information on the primary complaint and current symptoms, and information to be provided only 'if asked' by the tested-GP or symptom assessment app

Table 1 Triage levels assigned to each clinical vignette

\begin{tabular}{ll}
\hline Level of urgency advice & Description \\
\hline 1 & Call ambulance \\
2 & Go to emergency department \\
3 & See primary care within 4 hours \\
4 & See primary care same day \\
6 & See primary care non-urgent \\
\hline
\end{tabular}

(see online supplemental table 3). Each vignette was created with a list of gold standard correct conditions, arrived at through the majority decision of the vignette creation panel. This list included a main diagnosis and a list of other differential diagnoses (generally between one and four, but length-varying per vignette, as appropriate to the clinical history).

\section{Vignette review}

The vignettes were reviewed externally by a panel of three experienced primary care practitioners, each with more than 20 years primary care experience (see acknowledgements), recruited from the professional network of JC. The role of the review panel was to make changes to improve quality and clarity, and to set the gold-standard main diagnosis and differential diagnoses; this was determined by the majority view.

The gold-standard triage level was set independently of vignette creation, vignette review and vignette diagnosis gold-standard setting - this was done by a separate panel of three experienced primary care practitioners using a tie-breaker panel method based on the matching process set out by. ${ }^{6}$ The gold-standard optimal triage was assigned by the panel to a six-point scale (see table 1), independent of the native levels of urgency advice of any of the eight apps. The tested-GPs' triage and the levels of urgency advice of each app were mapped to this scale using the linear mapping set out in online supplemental figure 1.

\section{Assessment of apps and GPs using vignettes}

Seven external GPs were tested with the vignettes (the 'tested-GPs'), providing condition suggestions (preliminary diagnoses) for the clinical vignettes after telephone consultations with JC, who had the role of 'patient-actor'physician. All tested GPs were listed on the GP Register and licensed to practice by the UK General Medical Council and had an average of 11.2 years clinical experience post qualification as a doctor and 5.3 years post qualification as a GP. The seven GPs were recruited from the professional networks of $\mathrm{AB}, \mathrm{SU}$ and JC. Of these, four had previously worked for the Ada Health telehealth service Dr Chat but were no longer employees at Ada. This prior employment did not include any involvement in the development of the Ada symptom assessment app. The other three GPs had no employment connection to any of the app manufacturers. Five of the tested GPs completed telephone consultations for all 200 clinical-vignettes. One 
GP completed 130 telephone consultations but had to withdraw due to personal reasons. Another GP completed 100 telephone consultations but had to withdraw due to work commitments. Based on the information provided in the telephone consultation, the GPs were asked to provide a main diagnosis, up to five other differential diagnoses, and a single triage level (appropriate to a telephone triage setting).

\section{Assessment of vignettes by the symptom assessment apps and 'coverage'}

The clinical vignettes were entered into eight symptom assessment apps by eight primary care physicians playing the role of 'patient'-(app-entry-Dr-1 to -8 in figure 1). The versions of the symptom assessment mobile apps assessed were the most up to date version available for iOS download between the dates of 19 November 2019 and 9 December 2019. The version of the Buoy online symptom assessment tool used was the version available online between the dates of 19 November 2019 and 16 December 2019. The symptom assessment apps investigated were Ada, Babylon, Buoy, K Health, Mediktor, Symptomate, WebMD, Your.MD (see online supplemental table 4 for a description of these apps). The eight physicians were recruited from the professional network of $\mathrm{AB}, \mathrm{FP}$ and SU. They were listed on the GP Register and licensed to practice by the UK General Medical Council, with at least 2 years of experience as a GP and had never worked or consulted for Ada Health; these physicians had no other role in this study. Each physician entered 50 randomly assigned vignettes (out of 200) into each of four randomly assigned symptom assessment apps. If the app did not allow entry of the clinical vignette (lack of coverage), the reason for this was recorded, as was the reason for every vignette for which condition suggestions or levels of urgency advice were not provided. If entry was permitted, the physician recorded the symptom assessment app's condition suggestions and levels of urgency advice and saved screenshots of the app's results to allow for source data verification. In this way, each vignette was entered once in each app, with four physicians entering vignettes in each app.

\section{Source data verification}

Source data verification was carried out ( $100 \%$ of screenshots compared with spreadsheet data) and any missing or inaccurately transcribed data in the spreadsheets was quantified, recorded in this report and corrected to reflect the screenshot data.

\section{Metrics for assessing condition-suggestion accuracy}

We compared the top-1 suggested condition (M1), the top-3 suggested conditions (M3), and the top-5 suggested conditions (M5) provided by the seven tested GPs and the eight apps to the gold-standard main diagnosis. We also calculated the comprehensiveness and relevance of each GP's and each app's suggestions ${ }^{13}$ — see table 2 for a description of the metrics used for comparing conditionsuggestion accuracy.

\section{Assigning matches between tested-GPs/apps and the gold- standard}

Every suggested condition from the tested GPs and the apps was submitted anonymously to an independent panel of experienced primary care physicians who were recruited from the professional network of FP, and who were listed on the GP Register and licensed to practice by the UK General Medical Council, with at least 2 years of experience as a GP and had never worked or consulted for Ada Health. The panel had the role of deciding if the suggested condition matched the gold-standard diagnoses list, unless there was an explicit exact match-that is, identical text of the answer from the tested-GP/app and the gold standard. Matching was decided using a tiebreaker panel method which was based on the method set out by. ${ }^{6}$ The panel was presented with the condition suggestions blinded to their source. Panellists were instructed to use their own clinical judgement in interpreting whether condition suggestions were matches to

Table 2 Metrics used in comparison of condition-suggestion accuracy

\begin{tabular}{lll}
\hline Abbrev. & Full name & Description \\
\hline M1 (\%) & M1 (Matching-1) accuracy & $\begin{array}{l}\% \text { of cases where the top-1 condition-suggestion matches the gold-standard main } \\
\text { diagnosis. }\end{array}$ \\
\hline M3 (\%) & M3 (Matching-3) accuracy & $\begin{array}{l}\text { \% of cases where the top-3 condition-suggestions contain the gold-standard main } \\
\text { diagnosis. }\end{array}$ \\
\hline M5 (\%) & M5 (Matching-5) accuracy & $\begin{array}{l}{ }^{7} \text { of cases where the top-5 condition-suggestions contain the gold-standard main } \\
\text { diagnosis }\end{array}$ \\
COMP (\%) & Comprehensiveness & $\begin{array}{l}\text { Ratio of the (number of gold standard differentials matched by the suggested differentials) } \\
\text { to the (number of gold standard differentials for the vignette), expressed as a mean across } \\
\text { all vignettes. }{ }^{13}\end{array}$ \\
\hline RELE (\%) & Relevance & $\begin{array}{l}\text { Ratio of the (number of the suggested differentials that match with any of the gold } \\
\text { standard differentials for the vignette) to the(number of differentials provided by the tested- } \\
\text { GP or the symptom assessment app for the vignette), expressed as a mean across all } \\
\text { vignettes. }{ }^{13}\end{array}$ \\
\hline
\end{tabular}


the gold standard, supported by matching criteria (see online supplemental table 5).

\section{Mapping and comparing levels of urgency advice}

Triage suggestions from each GP and levels of urgency advice from each app were mapped to the gold standard triage levels using the simple linear mapping scheme set out in online supplemental figure 1 . The degree of deviation of GP triage urgency and of app levels of urgency advice was compared by reporting the percentage of vignettes for which GPs and symptom assessment apps were: (1) overconservative; (2) overconservative but suitable (one level too high); (3) exactly-matched; (4) safe but underconservative (one level too low); or, (5) potentially unsafe.

The WebMD assessment report only provides information on whether each suggested condition is urgent (via an urgency 'flag'). Finer urgency advice on each condition suggestion is available by clicking through to a separate detailed screen on each suggested condition, but unlike the other apps, no overall vignette-level summary urgency advice is provided. Meaningful comparison to the other apps or tested GPs was therefore not possible and WebMD was excluded from the urgency advice analysis in this study. For each app, with the exception of WebMD, the proportion of 'safe' urgency advice, is defined as advice at the gold standard advice level, more conservative, or no more than one level less conservative.

We used confusion matrices in order to fully visualise the severity of misclassification of advice levels. ${ }^{14}$ These confusion matrices were weighted in order to represent the relative seriousness of inappropriate urgency advice, either in the direction of being overly conservative (eg, inefficient use of healthcare system resources), or in the direction of being insufficiently conservative (potentially unsafe advice). The weighted confusion matrices were normalised to correct to the number of vignettes for which urgency advice were provided by each app and tested-GP.

\section{Statistical methods}

M1, M3 and M5 performance as well as levels of urgency advice were compared using descriptive statistics and tests appropriate for categorical data. $\chi^{2}$ tests were used to test whether the proportion of correct answers from all apps and from all tested GPs were drawn from the same distribution. In case of a significant difference, two-sided post hoc pairwise Fisher's exact tests ${ }^{1516}$ were used to compare individual app or tested-GP performances. Comprehensiveness and relevance (COMP and RELE) were assessed by Kruskal-Wallis-H-Test (KW-H-Test) applied to all 15 answer datasets (8 apps and 7 tested GPs), followed by post hoc pairwise testing using the two-sided Dunn test, ${ }^{15}$ in cases where there was a significant difference on the KW-H-Test. P values were corrected for multiple comparisons using the Benjamini-Hochberg procedure ${ }^{17}$ and considered significant if less than 0.05 . In figures, error bars for individual app and tested-GP performance represent $95 \%$ CI. These were calculated using the Wilson-Score method for categorical data (M1, M3 and M5) ${ }^{18}$ and using the percentile bootstrap method for COMP and RELE. ${ }^{19}$ The mean app and tested-GP scores were calculated as arithmetic means of the M1, M3, M5, COMP and RELE performance for each app and each tested GP, with error bars that represent the SD.

\section{Patient and public involvement}

Patients were not involved in setting the research questions, the design, outcome measures or implementation of the study. They were not asked to advise on interpretation or writing up of results. No patients were advised on dissemination of the study or its main results.

\section{RESULTS}

\section{Source data verification}

For vignette cases where the app-entry-Drs made data recording errors, these were corrected to match the source verification data saved in the screenshots. Full sets of screenshots were recorded by seven of the eight app-entry-Drs. One app-entry-Dr (\#4) did not record all screenshots for K Health, WebMD and for Your.MD and for this reason a subanalysis of the 150 vignettes for which full verification was possible for these apps is provided in online supplemental table 6 and 7. The differences in performance in this subanalysis is relatively minor and might be due to random differences between the 150 and full vignette sets or be due to app-entry-Dr-4 recording error.

\section{App coverage}

The apps varied in the proportion of vignettes for which they provided any condition suggestions (see figure 2, online supplemental tables 8-10). The reasons that some apps did not provide condition suggestions included: (1) not included in the apps' regulatory 'Intended Use' or another product design reason (eg, users below a set age limit, or pregnant users); (2) not suggesting conditions for users with severe symptoms (or possible conditions); (3) presenting problem not recognised by the app (even after rewording and use of synonyms); and, (4) some apps did not have coverage for certain medical specialties, for example, mental health. For $12 \%$ of the vignettes, the urgency advice from for $\mathrm{K}$ Health was not recorded due to app-entry-Dr-4 recording error and was not recorded in source verification data saved in the screenshots. The missing data is labelled in figure 2 and in the later figures describing the appropriateness of urgency advice. A subanalysis of the 150 vignettes for which full data and full verification was possible for $\mathrm{K}$ Health is provided in online supplemental table 6 .

\section{Suggested conditions: the 'required-answer' approach}

The approach adopted in other vignettes studies by authors in refs, semigran HL et $a l^{3}{ }^{36}$ Bisson LJ et $a l^{20}$ Burgess M et al, ${ }^{21}$ Powley L et $a l^{22}$ Pulse Today et $a l^{23}$ 


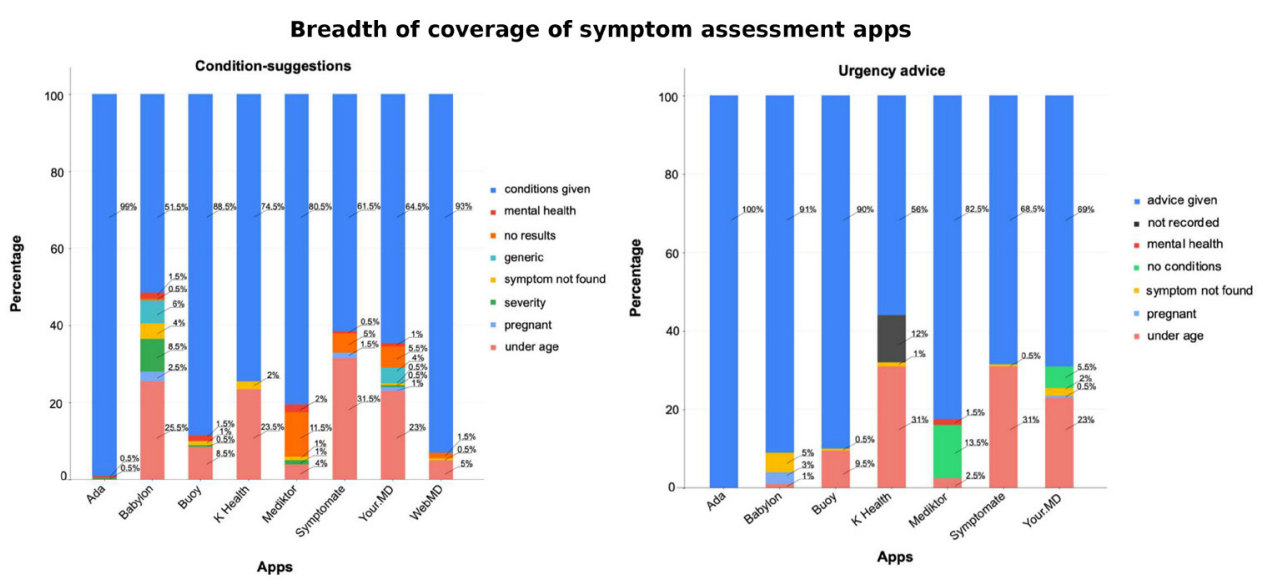

Figure 2 App breadth of coverage - that is, the proportion of vignettes for which condition suggestions and levels of urgency advice were provided. When condition suggestions or urgency were not provided, the principal reason for this is shown, or alternatively 'no results' when no reason was given. conditions given-condition suggestions were provided by the app; mental health - mental health vignettes where no condition suggestions/urgency advice was provided; no results - the app provided a clear statement that no condition suggestion results were found for the vignette (the reason why the app failed to give a condition suggestion for these vignettes is uncertain, but generally these vignettes relate to minor conditions, and in most cases it seems that the app does not have a matching condition modelled); generic-the app gave a generic answer rather than a condition, for example, 'further assessment is needed'; symptom not found-a directly or appropriately matching symptom to the presenting complaint could not be found in the app so the vignette could not be entered; severity - the app did not to give condition-suggestions for very serious symptoms - for example, the app stated only 'Condition causing severe (symptom)'; pregnant,vignettes for which no condition-suggestions/urgency advice was provided by the app as the patient was pregnant; under age-vignettes for which no condition suggestions/urgency advice was provided by the app as the patient was under its specified age limit; advice given -level of urgency advice was provided by the app; not recorded-one app-entry-Dr (\#4) did not fully record the levels of urgency advice, and there were no corresponding source data verification screenshots for this subset of data (see online supplemental table 6 for a subanalysis of the 150 vignettes with complete source-data-verified data for $\mathrm{K}$ Health on levels of urgency advice); no conditions - no condition suggestions were provided by the app, and, as a result of this, the app did not provide urgency advice. See online supplemental tables 8-10 for details.

Nateqi $\mathrm{J}$ et $a l^{24}$ has been to determine the percentage of all vignettes for which the app (or tested GP) provided an appropriate condition-suggestion-here, this analysis method is referred to as the 'required-answer' approach. Results are shown in figure 3. For a full description for each metric, see table 2.

\section{Suggested conditions: the 'provided-answer' approach}

For users or physicians choosing or recommending a symptom assessment app, it is relevant to know not only the app accuracy, but also how wide is its coverage and therefore the 'required-answer' analysis in the previous section is the most relevant analysis. An alternative approach is the provided-answer analysis, which is the number of correct suggested conditions provided by an app for each vignette for which it provides an answer. In other words, there was no penalty for an app that, for any reason, does not provide condition suggestions for a vignette, for example, children under 2 years old (see online supplemental tables 4 and 10). Both analyses are provided in this study in order to give a fully balanced overview of the performance of all the apps. The results for the provided-answer analysis are shown in figure 4 . For a full description for each metric, see table 2.

\section{Levels of urgency advice}

The urgency advice performance of each app is summarised in table 3. Tested GPs had safe triage performance of $97.0 \% \pm 2.5 \%$ (where safe is here defined as maximum one level less conservative than goldstandard, expressed per vignette provided with advice) three apps had safety performance within 1 SD of GPs (mean)—Ada: 97.0\%; Babylon: 95.1\%; and, Symptomate: $97.8 \%$. One app had a safety performance within 2 SDs of GPs-Your.MD*: 92.6\%. Three apps had a safety performance outside 2 SDs of GPs-Buoy: $80.0 \%(\mathrm{p}<0.001)$; K Health*: $81.3 \%(\mathrm{p}<0.001)$; Mediktor: $87.3 \%\left(\mathrm{p}=1.3 \times 10^{-3}\right)$ (*-for two of these apps one app-entry-Dr (\#4) did not record all screenshots needed for source data verification-see online supplemental table 6 for a subanalysis of fully verified data, which shows the same trend of results and no significant difference to the data recorded here).

Figure 5 summarises and compares urgency advice performance, including the proportion of vignettes for which some apps did not provide advice.

The visualisation in figure 5 provides a high-level overview of urgency advice performance; however, a limitation of this approach is that the full range of comparisons between gold standard triage and levels of urgency advice is not shown. The full range of overconservative and potentially unsafe urgency advice provided by each app and tested GP is shown in the weighted confusion matrices (figure 6). Low numbers in the matrices (coloured green and yellow) correspond to good urgency advice allocation, high numbers (coloured orange and red) correspond to 

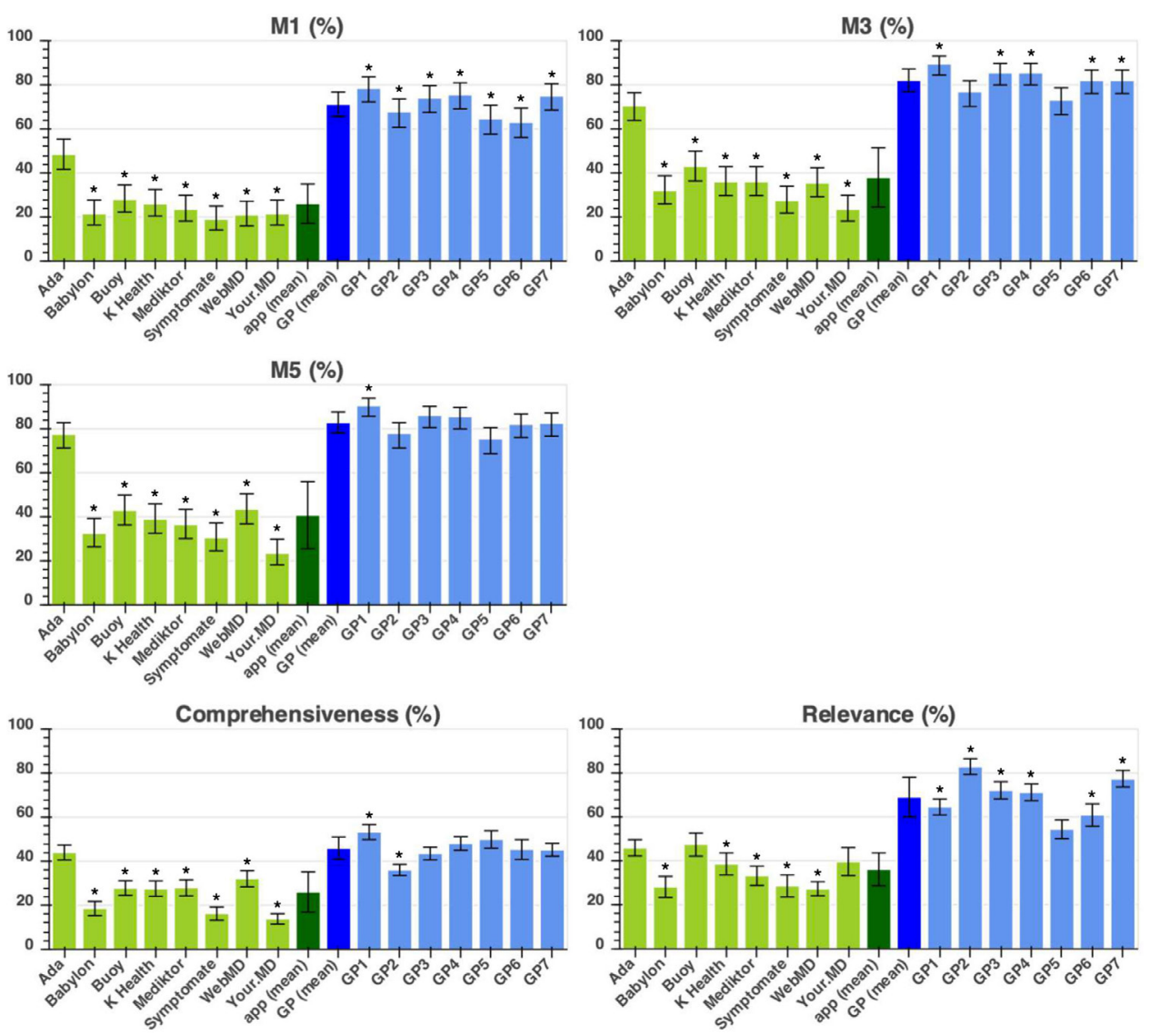

Figure 3 Required answer approach showing the performance metrics (M1, M3, M5, comprehensiveness and relevance-as defined in table 2) of the eight apps and seven tested general practitioners (GPs). App performance is coloured in light green, average (mean) app performance is in dark green, average (mean) tested-GP performance in dark blue, and individual testedGP performance in light blue. Statistical significance of the difference between the app with highest performance and all other apps/tested GPs is shown with the * symbol indicating: $p<0.05$. For one of these apps (Your.MD), one app-entry-Dr (\#4) did not record all screenshots needed for source data verification-see online supplemental table 7 for a subanalysis of fully verified data, which shows the same trend of results and no significant difference to the data recorded here.

bad urgency advice allocation. In order to visualise the overall urgency advice performance of each app, that is, performance both in urgency advice coverage and in the percentage of safe advice, these measures are plotted against each other in figure 7 .

\section{Subanalysis of performance in the NHS-derived and non-NHS- derived vignettes}

This study evaluated app and GP performance using 200 vignettes, of which $32.0 \%$ were derived from NHS Direct cases and $68.0 \%$ were created by the vignette creation team. The performance of each app and average GP performance stratified by vignette source (NHS or nonNHS derived) are shown in online supplemental table 11. The GPs and all apps performed better in providing appropriate urgency advice in the non-NHS vignettes than in the NHS-derived vignettes. In condition-suggestion accuracy, all GPs performed substantially better in M1, M3 and M5 for the non-NHS vignettes (differences in GP mean performance were $15.0 \%, 11.7 \%$ and $10.8 \%$, respectively). Differences in GP performance in COMP and RELE were not large, and performance in COMP was better (difference 3.1\%) in the NHS-derived vignettes.
Apps differed in their relative condition-suggestion accuracy between the NHS and non-NHS derived vignettes. Ada and Buoy, following the pattern of the GPs, performed substantially better in the non-NHS vignettes, while Symptomate performed similarly in both vignettes sets, and $\mathrm{K}$ Health, WebMD and Your.MD performance was relatively better in some and relatively weaker in other metrics in the two sets of vignettes. Mediktor was moderately better in the NHS derived vignettes for all metrics except RELE.

\section{DISCUSSION \\ Principal findings}

In this clinical vignette comparison of symptom assessment apps and GPs, we found that apps varied substantially in coverage, appropriateness of urgency advice and accuracy of suggested conditions.

Synthesising the analyses on the appropriateness of urgency advice (see table 3 and figures 5-7), the apps can be categorised as follows:

1. Levels of safe urgency advice within one SD from the average of GPs and: 

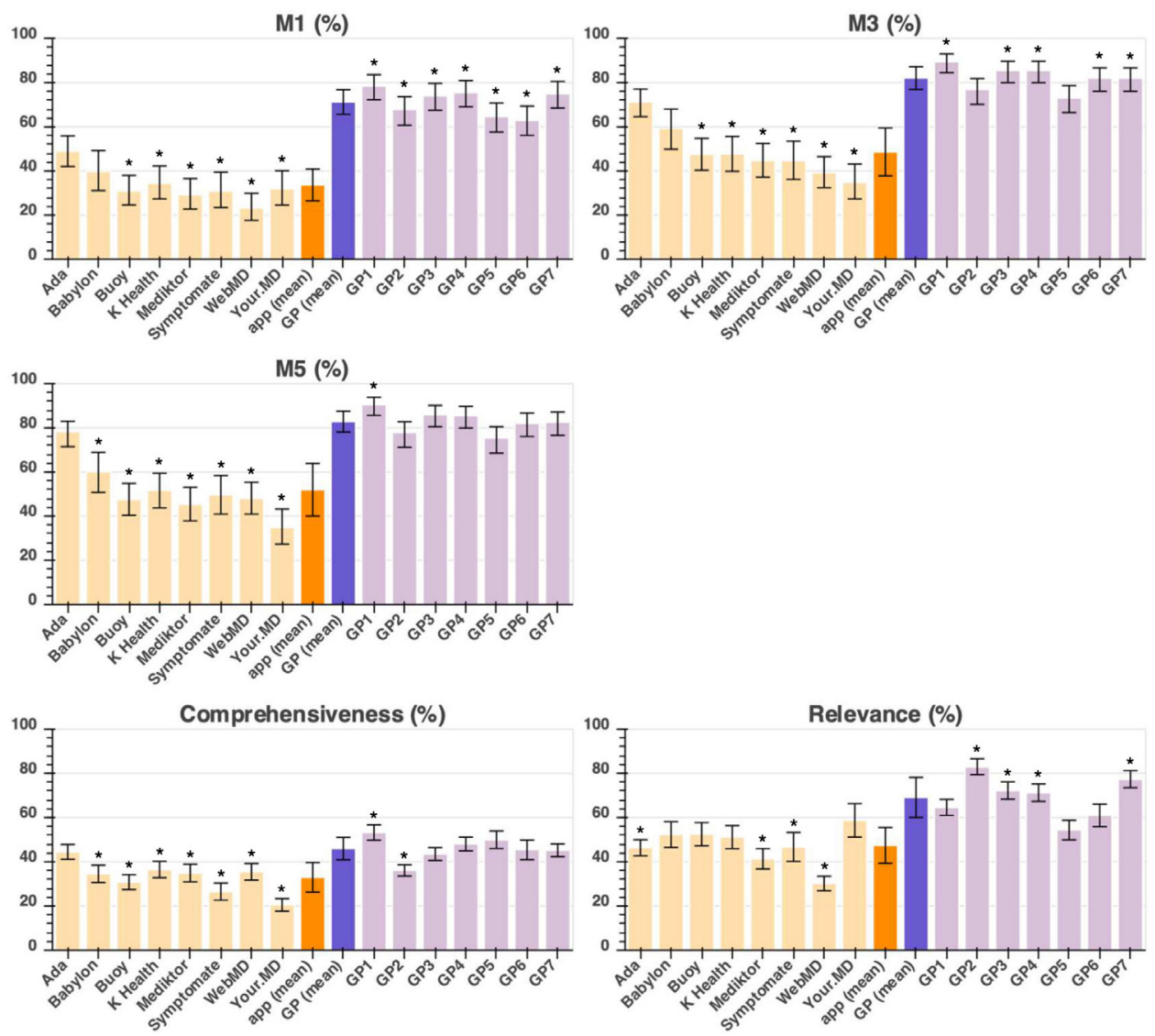

Figure 4 Provided-answer approach showing the performance metrics (M1, M3, M5, comprehensiveness and relevance-as defined in table 2) of the eight apps and seven tested general practitioners (GPs). App performance is coloured in light orange, average (mean) app performance is in dark orange, average (mean) tested-GP performance in dark purple, and individual tested-GP performance in light purple. Statistical significance of the difference between the app with highest performance and all other apps/tested GPs is shown with the * symbol indicating: $\mathrm{p}<0.05$. For one of these apps (Your.MD), one app-entry- $\operatorname{Dr}(\# 4)$ did not record all screenshots needed for source data verification - see online supplemental table 7 for a subanalysis of fully verified data, which shows the same trend of results and no significant difference to the data recorded here.

1a. Full-full or near-full coverage: Ada.

1b. Moderate coverage: Babylon.

1c. Low coverage: Symptomate.

2. Levels of safe urgency advice between one and two SD from the average of GPs and:

2a. Low coverage: Your.MD.

3. Levels of safe urgency advice below three SD from the average of GPs and:

3a. Moderate coverage: Buoy, Mediktor.

3b. Low coverage: K Health.

Condition suggestion coverage varies greatly with a range of $47.5 \%$ from highest (Ada; $99.0 \%$ ) to lowest (Babylon, 51.5\%). Although there is no absolute cut-off of what an acceptable condition suggestion coverage is, an app that can provide high coverage along with a high accuracy of condition suggestion and high urgency advice appropriateness, will generally be superior to an app with narrow coverage. There is no identifiable correlation between app M1 or M3 condition-suggestion accuracy or urgency-advice accuracy and the condition-suggestion coverage or urgency-advice coverage.

There was considerable variation in conditionsuggestion accuracy between the GPs and between apps.
For top-1 condition suggestion (M1), the range of tested GPs was $16.0 \%$, the SD 5.6\% and for M3 the range was $15.9 \%$ and SD 5.2\%. For the apps, the M1 conditionsuggestion accuracy range was $29.5 \%$, the SD $8.9 \%$ and the M3 range was $47.0 \%$ and SD $13.5 \%$. The GPs all outperformed apps for top-1 condition matching. For M3 and M5 (ie, including the gold standard diagnosis in top-3 and top-5 suggestions), the best performing app (Ada) was comparable to tested GPs, with no significant difference between its performance and the performance of several of the tested GPs. The top performing symptom assessment app (Ada) had an M3 27.5\% higher than the next best performing app (Buoy, $\mathrm{p}<0.001)$ and $47.0 \%$ higher than the worst-performing app (Your.MD, $\mathrm{p}<0.001)$. There was a significant difference between the top performing app (Ada) and other apps for all condition accuracy measures, with two exceptions for relevance (in the required-answer analysis).

There was also considerable variation in urgency advice performance between the GPs and between apps. The range of tested-GP safe advice was $6.0 \%$ and the SD was $2.5 \%$; for the apps, the range of safe advice was $17.8 \%$ and the SD 7.4\%. Tested GPs had an average safe advice 
Table 3 Triage levels assigned to each clinical-vignette, where safe is defined as maximum one level less conservative than gold-standard, expressed per vignette provided with advice.

\begin{tabular}{lcl}
\hline App/ tested GP & $\begin{array}{l}\text { Percentage of } \\
\text { safe advice }\end{array}$ & $\begin{array}{l}\text { P value (difference } \\
\text { to GP mean) }\end{array}$ \\
\hline Ada & 97.0 & NS \\
Babylon & 95.1 & NS \\
Buoy & 80.0 & $<0.001^{*}$ \\
K Health & 81.3 & $<0.001^{*}$ \\
\hline Mediktor & 87.3 & $1.3 \times 10^{-3 *}$ \\
Symptomate & 97.8 & NS \\
Your.MD & 92.6 & NS \\
\hline App mean \pm SD. & $90.1 \pm 7.4$ & - \\
\hline GP mean \pm SD. & $97.0 \pm 2.5$ & - \\
GP1 & 96.0 & NS \\
GP2 & 96.9 & NS \\
GP3 & 94.0 & NS \\
GP4 & 99.0 & NS \\
\hline GP5 & 100.0 & NS \\
GP6 & 93.9 & NS \\
GP7 & 99.5 & NS \\
\hline
\end{tabular}

${ }^{*} \mathrm{P}<0.05$. For two of these apps (K Health \& Your.MD), one appentry-Dr (\#4) did not record all screenshots needed for source data verification-see online supplemental table 6 for a subanalysis of fully verified data, which shows the same trend of results and no significant difference to the data recorded here). This analysis is for those vignettes for which urgency advice was provided (ie, a 'provided answer) analysis.

GP, general practitioner; NS, no significant difference.

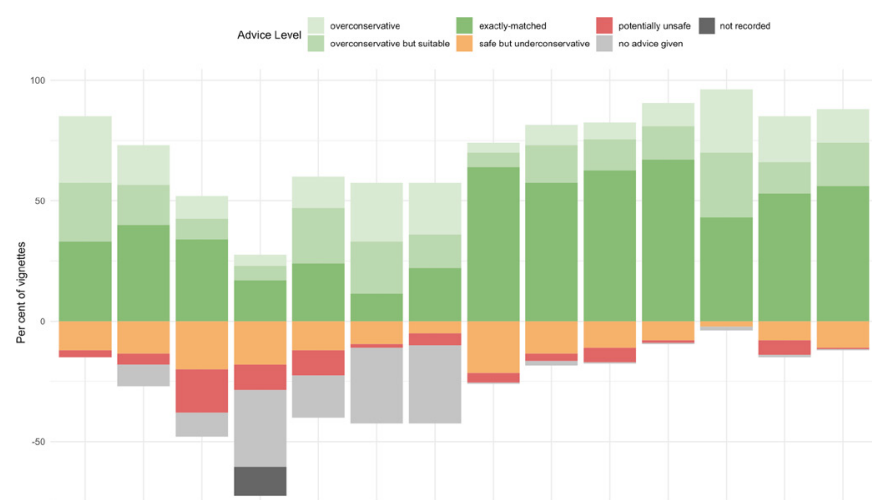

Figure 5 Accuracy of urgency advice displayed as a stacked bar chart centred on the gold standard triage. For two of these apps (K Health \& Your.MD), one app-entryDr (\#4) did not record all screenshots needed for source data verification - see online supplemental table 6 for a subanalysis of fully verified data, which shows the same trend of results and no significant difference to the data recorded here. GP, general practitioner. performance of $97.1 \pm 2.5 \%$ and only three apps had safe advice performance within $1 \mathrm{SD}$ of the GPs (mean) Ada: 97.0\%; Babylon: $95.1 \%$; and Symptomate: $97.8 \%$.

The results support acceptance of the hypothesis 1 (a), that GPs have better performance than the apps on condition-suggestion accuracy. Hypothesis 1 (b) and 1 (c) were that GPs would have better performance than the apps in the appropriateness and safety of urgency advice, and these hypotheses are partially rejected, as, while overall GPs performed better in urgency advice than apps, some individual apps performed as well as GPs in urgency advice safety and similarly to GPs in urgency advice accuracy. Hypothesis 2 was that performance of each app would be consistent across the three metrics (condition-suggestion accuracy, appropriateness and safety of urgency advice), and this hypothesis is rejected as the results showed that apps performing well in urgency advice safety or appropriateness did not necessarily have high condition-suggestion accuracy. Hypothesis 3, that apps would differ from one another in their performance across the three metrics. This hypothesis is accepted as there were major differences between apps in all three metrics.

There were relative differences in the performance of the GPs and of the apps in the NHS-derived and nonNHS derived vignettes; however, the overall conclusions of this study are valid for both sets of vignettes, and the performance of each app evaluated is broadly similar irrespective of whether all vignettes are considered or the NHS-derived or non-NHS-derived subsets. The differences in performance likely reflect differences in the case structure complexity in the vignettes, the degree of ambiguity in the vignettes, the individual question flow of the apps, differences in condition coverage of the apps and the different frequencies of disease categories in the vignettes-for example, there were more cardiovascular disease cases in the NHS-derived vignettes, 7/64 (10.9\%) compared with $7 / 136(5.1 \%)$ in the non-NHS-derived vignettes.

\section{Strengths and limitations of this study}

The systematic review of Chambers et at identified limitations of published studies on the safety and accuracy of symptom assessment apps as: (1) not being based on real patient data; (2) not describing differences in outcomes between symptom assessment apps and health professionals; (3) covering only a limited range of conditions; (4) covering only uncomplicated vignettes; and (5) sampling a young healthy population not representative of the general population of users of the urgent care system. Of these limitations, only one applies to this study - the limitation of being based on clinical vignettes rather than on real-patient data. The effect of this limitation has been minimised through the development of many of the vignettes to be highly realistic through the use of anonymised real patient data collated from NHS Direct transcripts. The use of real patient data with an actual diagnosis is not without 


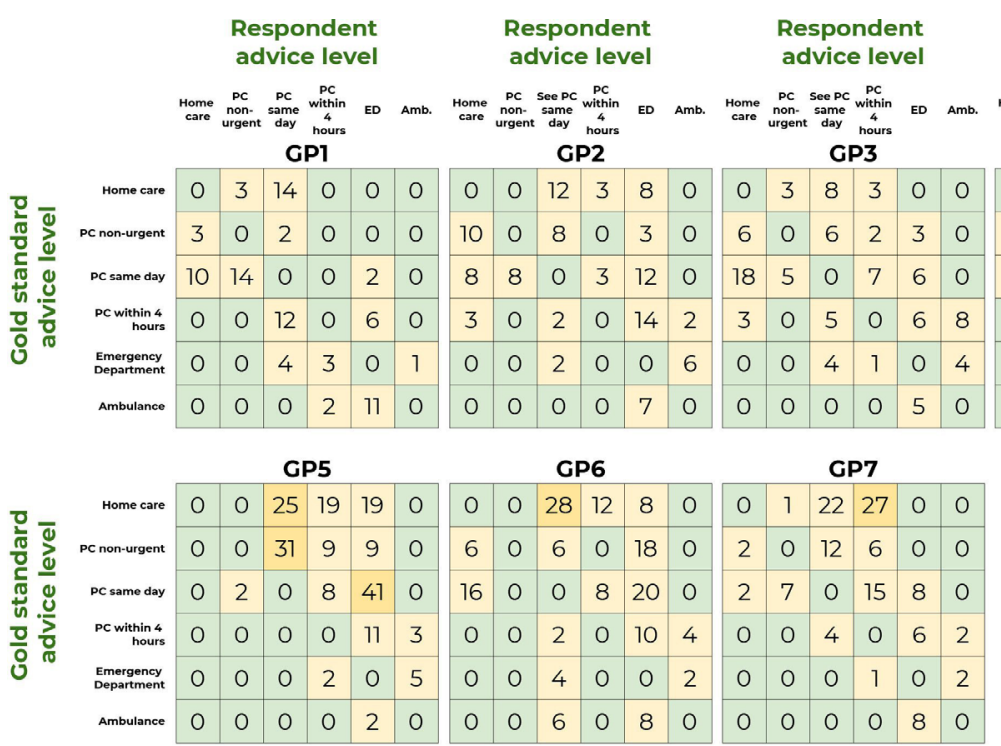

\begin{tabular}{|c|c|c|c|c|c|c|c|c|c|c|c|c|c|c|c|c|c|c|c|c|c|c|c|c|}
\hline & \multicolumn{6}{|c|}{ Ada } & \multicolumn{6}{|c|}{ Babylon } & \multicolumn{6}{|c|}{ Buoy } & \multicolumn{6}{|c|}{ K Health } \\
\hline$\delta_{-}$ & 0 & 6 & 0 & 27 & 16 & 5 & 0 & 7 & 13 & 7 & 4 & 0 & 0 & 2 & 9 & 0 & 9 & 0 & 0 & 11 & 0 & 0 & 14 & 0 \\
\hline PC non-urgent & 5 & 0 & 0 & 20 & 9 & 4 & 5 & 0 & 14 & 4 & 1 & 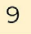 & 14 & 0 & 12 & 4 & 7 & 4 & 7 & 0 & 0 & 0 & 16 & 0 \\
\hline PC same day & 10 & 9 & 0 & 21 & 46 & 3 & 4 & 14 & 0 & 3 & 22 & 10 & 38 & 13 & 0 & 0 & 18 & 0 & 18 & 45 & 0 & 2 & 14 & 0 \\
\hline 응 $\frac{5}{0}$ & 3 & 0 & 0 & 0 & 15 & 6 & 7 & 0 & 7 & $\mathrm{O}$ & 8 & 11 & 13 & 7 & 3 & 0 & 3 & 0 & 0 & 14 & $\mathrm{O}$ & 0 & 7 & 0 \\
\hline $\begin{array}{l}\text { Emergenc, } \\
\text { Departmer }\end{array}$ & 0 & 0 & 0 & 2 & 0 & 7 & 4 & 0 & 4 & 0 & 0 & 4 & 13 & 3 & 9 & 2 & 0 & 1 & 14 & 27 & 0 & 2 & 0 & 2 \\
\hline Ambulan & 0 & 0 & 0 & 0 & 8 & 0 & 0 & 0 & 3 & 2 & 3 & 0 & 0 & 4 & 7 & 2 & 11 & 0 & 0 & 36 & 0 & 0 & 11 & 0 \\
\hline
\end{tabular}
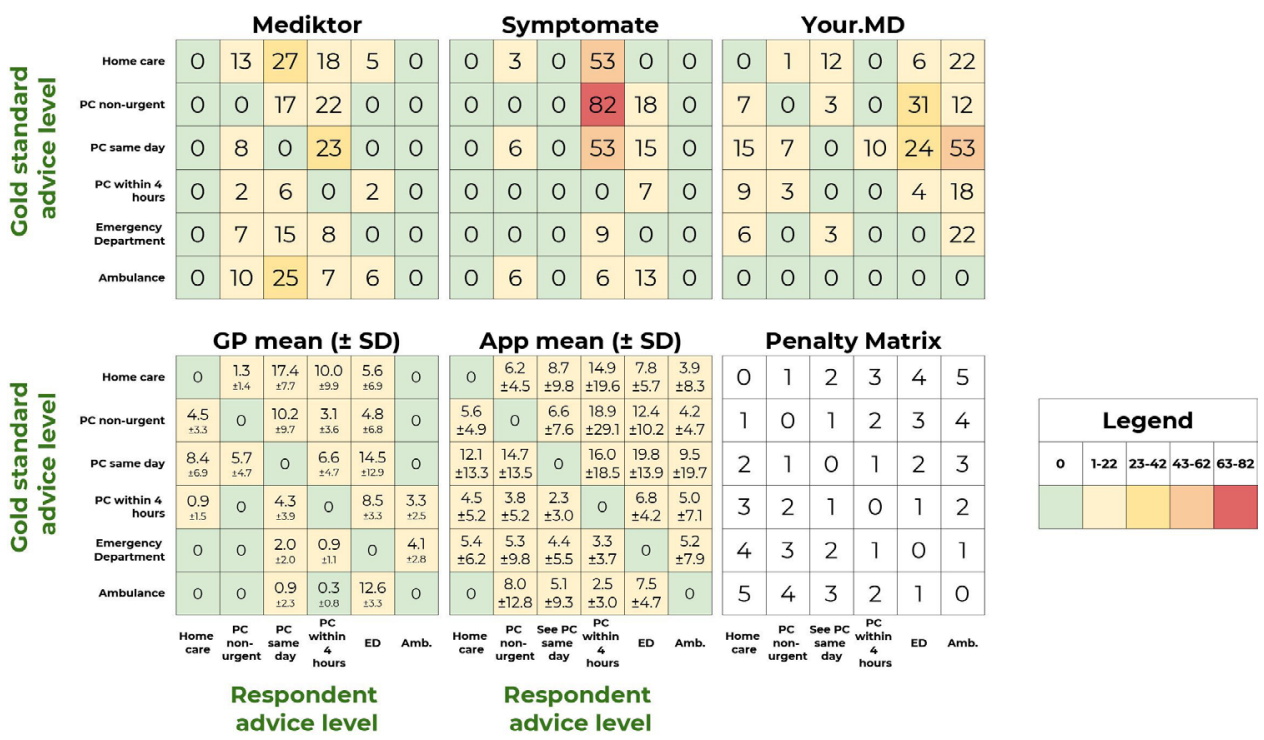

Figure 6 Weighted confusion matrices showing the detailed triage assignments for each app. For two of these apps (K Health \& Your.MD), one app-entry-Dr (\#4) did not record all screenshots needed for source data verification-see online supplemental table 6 for a subanalysis of fully verified data, which shows the same trend of results and no significant difference to the data recorded here. Amb, ambulance; ED, emergency department; GP, general practitioner; PC, primary care

its limitations in the evaluation of symptom assessment app accuracy as it relies on face-to-face consultation to confirm diagnosis. Very often diagnosis is only provided after physical examination or diagnostic tests, so comparison is confounded as the real patient diagnosis is based on additional information not made available to the app. The vignettes approach has allowed this study to be designed to minimise the limitations (2)-(5) identified by Chambers et al. ${ }^{4}$ This has been done for limitation (2) through inclusion of a 7-GP comparator group; for limitation (3) by development of vignettes for conditions spanning all body systems and sampling all medical specialisms relevant to primary care presentation; for limitation (4) by designing clinical vignettes including not-only simple and common situations, but also moderately complex and challenging presentations; for limitation (5) through including vignettes spanning from 1 month to 89 years old. 
Percentage of safe advice vs coverage

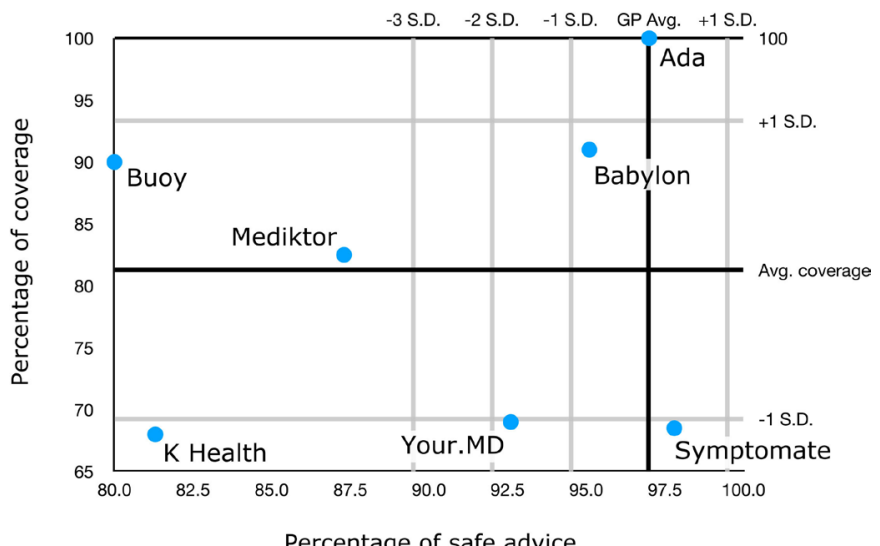

Figure 7 Summary plot of the urgency advice performance of each app. The urgency advice coverage of each app (with respect to app average) is plotted against the percentage of safe advice (with respect to the general practitioner (GP) average). For two of these apps (K Health \& Your.MD), one app-entry-Dr (\#4) did not record all screenshots needed for source data verification-see online supplemental table 6 for a subanalysis of fully verified data, which shows the same trend of results and no significant difference to the data recorded here.

Relative strengths of this study are the large number of clinical vignettes included $(n=200)$, along with the separation in the design of clinical-vignette writing from the process of deciding on the gold-standard main and secondary differential diagnoses and appropriate levels of urgency advice. Another strength of this study is that GPs were tested with vignettes in a manner that simulates real clinical consulting - in this way the GPs consultation process was assessed, enabling a fair comparison to the apps. Vignettes were entered into the apps by eight additional primary care physicians acting as the user (app-entry-Dr-1-8). A physician also 'acted' as the patient being assessed by the GPs in the phone consultations. It has been argued that lay-person entry is closest to the real intended use of symptom assessment $\mathrm{apps}^{25}$; however, it is known that lay-people are less reliable at entering clinical vignettes than healthcare providers. ${ }^{26} \mathrm{~A}$ further strength of this study is that each decision of whether a condition suggestion (from an app or a GP) matched the clinical-vignette's main and other differential diagnoses was made in a rigorous manner following the 3-physician tie-breaker panel approach of Semigran $e t a l^{6}$

A limitation of this study was that a systematic and comprehensive process was not used to select the symptom assessment apps to be included. Practical considerations in study design necessitated that the study evaluated a total of eight apps, due to the large number of vignettes assessed. The aim of the selection process was to include only apps with similar intended use, and to include those most used, those still in current use which have been evaluated in other studies, those most used within the UK as the study used vignettes based on UK patient data and those most used in the USA, as it is a highly important market for symptom assessment applications. Apps were then selected using a hybrid approach, including, based on the knowledge of available apps of the study team, internet searching and industry sources on usage data of symptom assessment apps. For apps identified using this approach, rigorous exclusion criteria were applied: all apps which did not provide, for a general population, primary care condition suggestions and urgency advice, were excluded. Through the application of this methodology, we have assured that all included apps were appropriate and relevant for inclusion, but it is possible, due to the limiting of the study to eight apps, without a rigorous prioritisation selection procedure, that there was unintentional bias in app inclusion. Nonetheless, the study included all the highest used symptom assessment apps in the UK and the USA, at the time of app selection, based on app usage statistics for the Google Play and Apple iOS app stores. The non-systematic selection criteria used were that, at the time of selection: (1) Babylon and Ada are leading symptom assessment smartphone apps in the UK; (2) K Health, WebMD and Ada are the most used in USA (usage data from Sensely, https:/ /www.sensely.com); (3) Mediktor and Buoy have existing published data ${ }^{727}$; and (4) Your.MD has a similar user experience and user interface to Babylon and Ada and has been compared with them in small non-peer reviewed studies. ${ }^{21} 23$

Direct comparison of levels of urgency advice between individual apps and between apps and GPs was challenging because (1) some apps provided no levels of urgency advice for large numbers of vignettes; (2) performing well in one level of urgency advice trades off performance in other levels of urgency advice; (3) the nature of urgency advice reporting was different in WebMD (see the Methods section).

Furthermore, the vignettes may have had a UK bias and some of the symptom assessment apps (eg, Buoy, $\mathrm{K}$ Health \& WebMD) are primarily used in the USA. The population demographics and the health conditions represented in the vignettes were broadly similar to demographics extracted from UK and NHS England health statistics (see the online supplemental Appendix S1, including online supplemental figures 2 and 3). Ada employees were involved in the vignette creation process, and although it was ensured that the vignette creation was separated from app medical intelligence development, unintentional bias could have resulted in vignette wording that was more accessible to symptom assessment apps than the average real word primary care clinical presentation is. A data acquisition error by one of the app-entry-Drs meant there were unrecorded urgency advice data for $12.0 \%$ of vignettes for one app (K Health) and incomplete source data verification screenshots for two other apps (Your.MD and WebMD). The implication for this for the main analysis was investigated in two subanalyses in the data supplement. Future studies could ensure ongoing source data verification rather than waiting until the end of study data collection for review. It 
is an unavoidable limitation that software evolves rapidly, and the performance of these apps may have changed significantly (for better or worse) since the time of data collection. Finally, this study was designed, conducted and disseminated by a team that includes employees of Ada Health; future research by independent researchers should seek to replicate these findings and/or develop methods to continually test symptom assessment apps.

\section{Comparisons to the wider literature}

The results of this study are qualitatively broadly similar to reported results from other interapp relative performance studies, including one peer-reviewed study ${ }^{24}$ and two non-peer-reviewed studies. ${ }^{21}{ }^{23}$ A peer-reviewed study using 45 ear, nose and throat (ENT)-vignettes ${ }^{24}$ evaluated M1 and M3 results and found that Ada had substantially better performance than other apps. Overall, Ada was the second-best performing app out of 24 tested apps in the ENT discipline. ${ }^{24}$

A small non-peer-reviewed independent clinical vignettes study tested NHS 111, Babylon, Ada and Your. MD and found similar overall results to this study ${ }^{23}$; they also found that all apps were successful at spotting serious conditions, such as a heart attack, and that they were fast and easy to use. A second small 2017 non-peerreviewed independent vignettes study, ${ }^{21}$ that was carried out by established symptom assessment app academic researchers, tested Babylon, Ada and Your.MD. The trend of the results was similar to those in this study.

In an observational study carried out in a Spanish ED waiting room, the Mediktor symptom assessment app was used for non-urgent emergency cases for patients above 18 years old. ${ }^{7}$ The study calculated accuracy with consideration only for those patients whose discharge diagnosis was modelled by the app at the time. For a total of 622 cases, Mediktor's M1 score was reported as $42.9 \%$, M3 score as $75.4 \%$ (ie, the symptom assessment app's top-1 (M1), top-3 (M3), or top-10 condition-suggestion(s) matched the discharge diagnosis in this percentage of cases). When Moreno Barriga et al reported results are refactored to consider all patient discharge diagnoses (the standard approach) the: M1 is $34.0 \%$ and M3 is $63.0 \%$, compared with M1 of $23.5 \%$ and M3 of $36.0 \%$ for Mediktor in this study (all-vignettes data). The reason for lower Mediktor performance in the current study compared with the study in Moreno Barriga $e t a l^{7}$ is not known but it may be related to a different range of conditions or difficulty level than the non-urgent emergency cases presenting to the ED-for example-the vignettes in this study contain many true emergency cases and also many GP or pharmacy/treat-at-home cases which would not be represented by the ED patients included in Moreno Barriga et $a l^{7}$. In 2017, a 42-vignette evaluation of WebMD ${ }^{28}$ determined its accuracy for ophthalmic condition suggestion: M1 was $26.0 \%$ and M3 was $38.0 \%$. Urgency advice based on the top diagnosis was appropriate in $39.0 \%$ of emergency cases and $88.0 \%$ of nonemergency cases.
The manufacturers of the apps Babylon and Your. MD responded to the two non-peer reviewed studies ${ }^{2123}$ observing that their apps have been updated and improved subsequent to the publication of those reports. Nevertheless, the findings with respect to condition-suggestion performance, in the later peer-reviewed study by Nateqi et $a l^{24}$ and in the present study appear to be in line with those from the two non-peer-reviewed studies.

\section{Implications for clinicians and policy-makers}

The results of this study are relevant for home users of symptom assessment apps, and to healthcare providers offering advice to their patients on which symptom assessment apps to choose. There are large (and statistically significant) differences between app coverage, suggested condition accuracy and urgency-advice accuracy. One of the biggest challenges in comparing symptom checker apps are the differences in coverage. Some coverage restrictions, such as not allowing symptom assessment for one user subgroup (eg, children), have no negative effect on the app's effective use for other user subgroups (eg, adults). Other situations, such as the inability to search for certain symptoms, providing no conditionsuggestions/urgency advice for certain input symptoms, or, excluding comorbidities, mental health or pregnancy are more problematic and can raise concerns about the safety and benefits of the app for users who might be in those groups.

\section{Unanswered questions and future research}

Future research should evaluate the performance of the apps compared with real-patient data-multiple separate single-app studies are a very unreliable way to determine the true level of the state of the art of symptom-assessment apps. A positive step in this direction is the ITU/WHO Focus Group AI for Health (FG-AI4H) through which several manufacturers of symptom assessment apps evaluated in this study are working collaboratively to create standardised app benchmarking with independently curated and globally representative datasets. ${ }^{29}$ Additional areas that could be explored in such studies are comparative economic impact, understanding user behaviour following an assessment, that is, compliance with urgency advice (extending the approach of Winn $e t a l^{27}$ ) and impact on health services usage, and, the impact of using the apps to complement a standard GP consult (eg, through diagnostic-decision support). While it has been argued that the accuracy of urgency advice may be the most important output from a health assessment app, the condition suggestions may be valuable to support patient decision-making. ${ }^{27}$ To address the effect of patients entering data directly into an app about their own acute conditions, an observational investigation is currently underway in an acute clinical setting in the USA by investigators including coauthors of this study. This includes a survey of users' technological literacy and user experience. 


\section{CONCLUSIONS}

This study provides useful insights into the relative performance of eight symptom-assessment apps, compared with each other and compared with seven tested GPs, in terms of their coverage, their suggested condition accuracy and the accuracy of their levels of urgency advice. The results show that the best performing of these apps have a high level of urgency advice accuracy which is close to that of GPs. Although not as accurate as GPs in top-1 suggestion of conditions, the best apps are close to GP performance in providing the correct condition in their top-3 and top-5 condition suggestions.

While no digital tool outperformed GPs in this analysis, some came close, and the nature of iterative improvements to software suggests that further improvements will occur with experience and additional evaluation studies.

The findings of this vignettes study on urgency advice are supportive of the use of those symptom-assessment apps, which have urgency advice safety similar to the levels achieved by GPs, in the use case of supplementing telephone triage (a use case described in Chambers $e t a t^{4}$ ). The findings are also indicative of the future potential of AI-based symptom assessment technology in diagnostic decision support; however, this is an area that requires specific clinical evidence and regulatory approval. Further studies, which include direct use of the symptom assessment apps by patients, are required to confirm clinical performance and safety.

\section{Acknowledgements Paul Taylor (UCL Institute of Health Informatics)} independently reviewed and made suggestions on the study protocol, and after study data collection was complete, reviewed and made suggestions in a draft of this manuscript with respect to the analysis approach and the study description. Vignette review was carried out by the following experienced primary care physicians: Alison Grey, Helen Whitworth, Jo Leahy. Study support was provided by Linda Cen (Data Engineer, Ada Health GmbH), Leif Ahlgrimm (Student IT System Administrator, Ada Health GmbH), Neil Rooney (Senior IT System Administrator, Ada Health GmbH). Henry Hoffmann provided helpful suggestions on the final manuscript.

Contributors SG, AM, CC, JC, HF, FP, ET, WV, NV \& CN contributed to the planning (study conception, protocol development). SG, AM, AB, CC, JC, FP, CR, SU, WV, $\mathrm{NV}, \& \mathrm{CN}$ contributed to the conduct (coordination of vignette creation, review, coordination of GP or app-testing, condition-matching panel coordination). SG, AM, CC, JC, HF, EM, MM, JM, FP, CR, ET, NV, PW \& CN contributed to the data analysis \& interpretation. SG, AM, JC, HF, JM, FP, CR, ET, VV, NV, PW \& CN contributed to the reporting (report writing). All the authors contributed to commenting on drafts of the report. SG is the guarantor for this work.

Funding This study was funded by Ada Health $\mathrm{GmbH}$. HF has not received any compensation from Ada Health financial or otherwise.

Competing interests All of the authors, with the exception of HF, are or were employees of, contractors for, or hold equity in the manufacturer of one of the tested apps (Ada Health GmbH). See author affiliations. SG, AM, AB, CC, EM, MM, JM, FP, ET, SU, NV and CN are employees or company directors of Ada Health GmbH and some of the listed hold stock options in the company. CR and V are former employees of Ada Health GmbH. JC and PW have or have had consultancy contracts with Ada Health $\mathrm{GmbH}$. The Ada Health $\mathrm{GmbH}$ research team has received research grant funding from Fondation Botnar and the Bill \& Melinda Gates Foundation. PW has received speaker fees from Bayer and honoraria from Roche, ARISLA, AMIA, IMI, $\mathrm{PSI}$, and the BMJ.

Patient consent for publication Not required.

Provenance and peer review Not commissioned; externally peer reviewed.
Data availability statement All data relevant to the study are included in the article or uploaded as supplementary information with the exception of the case vignettes. The vignettes used in this study can be made available on request to the corresponding author SG, provided that they will be used for genuine scientific purposes, and that these purposes will not compromise their utility in future assessment of symptom assessment applications (for example, by making them publicly available, and therefore accessible to the medical knowledge learning of symptom assessment app manufacturers). They are not publicly available due to planned periodic update of the study analysis, which will be carried out by Ada Health and other independent scientific researchers, in order to monitor comparative change in app performance over time. All vignette access requests will be reviewed and (if successful) granted by the Ada Health Data Governance Board. The vignettes will not be disclosed to the Ada medical intelligence team or to other app developers.

Supplemental material This content has been supplied by the author(s). It has not been vetted by BMJ Publishing Group Limited (BMJ) and may not have been peer-reviewed. Any opinions or recommendations discussed are solely those of the author(s) and are not endorsed by BMJ. BMJ disclaims all liability and responsibility arising from any reliance placed on the content. Where the content includes any translated material, BMJ does not warrant the accuracy and reliability of the translations (including but not limited to local regulations, clinical guidelines, terminology, drug names and drug dosages), and is not responsible for any error and/or omissions arising from translation and adaptation or otherwise.

Open access This is an open access article distributed in accordance with the Creative Commons Attribution Non Commercial (CC BY-NC 4.0) license, which permits others to distribute, remix, adapt, build upon this work non-commercially, and license their derivative works on different terms, provided the original work is properly cited, appropriate credit is given, any changes made indicated, and the use is non-commercial. See: http://creativecommons.org/licenses/by-nc/4.0/.

\section{ORCID iDs}

Stephen Gilbert http://orcid.org/0000-0002-1997-1689

Maryam Montazeri http://orcid.org/0000-0003-4688-9311

\section{REFERENCES}

1 McDaid D, Park A-L. Online health: untangling the web, 2011

2 Van Riel N, Auwerx K, Debbaut P, et al. The effect of Dr Google on doctor-patient encounters in primary care: a quantitative, observational, cross-sectional study. BJGP Open 2017;1:bjgpopen17X100833.

3 Semigran HL, Levine DM, Nundy S, et al. Comparison of physician and computer diagnostic accuracy. JAMA Intern Med 2016;176:1860-1.

4 Chambers D, Cantrell AJ, Johnson M, et al. Digital and online symptom checkers and health assessment/triage services for urgent health problems: systematic review. BMJ Open 2019;9:e027743.

5 Millenson ML, Baldwin JL, Zipperer L, et al. Beyond Dr. Google: the evidence on consumer-facing digital tools for diagnosis. Diagnosis 2018;5:95-105.

6 Semigran HL, Linder JA, Gidengil C, et al. Evaluation of symptom checkers for self diagnosis and triage: audit study. BMJ 2015;351:h3480.

7 Moreno Barriga E, Pueyo Ferrer I, Sánchez Sánchez M, et al. [A new artificial intelligence tool for assessing symptoms in patients seeking emergency department care: the Mediktor application]. Emergencias 2017;29:391-6.

8 Greenhalgh T, Wherton J, Shaw S, et al. Video consultations for covid-19. BMJ 2020;368:m998.

9 Heymann DL, Shindo N. Who scientific and technical Advisory group for infectious hazards COVID-19: what is next for public health? Lancet 2020;395:542-5.

10 Converse L, Barrett K, Rich E, et al. Methods of observing variations in physicians' decisions: the opportunities of clinical vignettes. $J$ Gen Intern Med 2015;30:586-94.

11 Evans SC, Roberts MC, Keeley JW, et al. Vignette methodologies for studying clinicians' decision-making: validity, utility, and application in ICD-11 field studies. Int J Clin Health Psychol 2015;15:160-70.

12 Veloski J, Tai S, Evans AS, et al. Clinical Vignette-Based surveys: a tool for assessing physician practice variation. Am J Med Qual 2005;20:151-7.

13 Berner ES, Webster GD, Shugerman AA, et al. Performance of four computer-based diagnostic systems. N Engl J Med 1994;330:1792-6. 
14 Swaminathan S, Qirko K, Smith T, et al. A machine learning approach to triaging patients with chronic obstructive pulmonary disease. PLoS One 2017;12:e0188532.

15 Nayak BK, Hazra A. How to choose the right statistical test? Indian J Ophthalmol 2011;59:85-6.

16 Shan G, Gerstenberger S. Fisher's exact approach for post hoc analysis of a chi-squared test. PLoS One 2017;12:e0188709.

17 Benjamini Y, Hochberg Y. Controlling the false discovery rate: a practical and powerful approach to multiple testing. Journal of the Royal Statistical Society: Series B 1995;57:289-300 https://www. jstor.org/stable/2346101?seq $=1$

18 Wilson EB. Probable inference, the law of succession, and statistical inference. J Am Stat Assoc 1927;22:209-12.

19 Efron B, Tibshirani RJ. An introduction to the bootstrap (Monographs on statistics and applied probability. New York: Chapman and Hall/ CRC, 1998.

20 Bisson LJ, Komm JT, Bernas GA, et al. How accurate are patients at diagnosing the cause of their knee pain with the help of a web-based symptom checker? Orthop J Sports Med 2016;4 doi:10.1177/2325967116630286

21 Burgess M. Can you really trust the medical apps on your phone? Wired UK, 2017. Available: https://www.wired.co.uk/article/healthapps-test-ada-yourmd-babylon-accuracy [Accessed 25 Mar 2020].
22 Powley L, Mcllroy G, Simons G, et al. Are online symptoms checkers useful for patients with inflammatory arthritis? BMC Musculoskelet Disord 2016;17:362.

23 Pulse Today. What happened when pulse tested symptom checker apps. Available: http://www.pulsetoday.co.uk/news/analysis/whathappened-when-pulse-tested-symptom-checker-apps/20039333. article [Accessed 25 Mar 2020].

24 Nateqi J, Lin S, Krobath H, et al. Vom Symptom zur Diagnose Tauglichkeit von symptom-checkern. HNO 2019;67:334-42.

25 Fraser $\mathrm{H}$, Coiera E, Wong D. Safety of patient-facing digital symptom checkers. Lancet 2018;392:2263-4.

26 Jungmann SM, Klan T, Kuhn S, et al. Accuracy of a Chatbot (ADA) in the diagnosis of mental disorders: comparative case study with lay and expert users. JMIR Form Res 2019;3:e13863.

27 Winn AN, Somai M, Fergestrom N, et al. Association of use of online symptom Checkers with patients' plans for seeking care. JAMA Netw Open 2019;2:e1918561.

28 Shen C, Nguyen M, Gregor A, et al. Accuracy of a popular online symptom checker for ophthalmic diagnoses. JAMA Ophthalmol 2019;137:690-2.

29 Wiegand T, Krishnamurthy R, Kuglitsch M, et al. WHO and ITU establish benchmarking process for artificial intelligence in health. Lancet 2019;394:9-11. 